\title{
Evaluación e intervención psicológica en jugadoras de hockey sobre hierba femenino
}

\section{Evaluation and psychological intervention in female grass hockey players}

\section{Avaliação e intervenção psicológica em jogadoras de hóquei em grama}

\author{
Tutte, V.1, Reche, C.2, Álvarez, V. 1 \\ (1)Facultad de Ciencias de la Salud. Universidad Católica del Uruguay. Montevideo-Uruguay. \\ (2)Facultad de Enfermería. Universidad Católica San Antonio de Murcia (UCAM).
}

\begin{abstract}
RESUMEN
El objeto de este estudio de caso fue evaluar las habilidades psicológicas de jugadoras de hockey hierba, y, por otro lado, la eficacia de la participación en un programa de entrenamiento psicológico en las características psicológicas relacionadas con el rendimiento deportivo. Participaron 10 jugadoras de hockey de 16 a 26 años de la selección de hockey hierba femenino de categoría senior. La intervención psicológica se realizó en Montevideo-Uruguay, durante 16 sesiones a través de trabajo individualizado (voluntario) y trabajo grupal (obligatorio) con un enfoque cognitivoconductual. La intervención estuvo formada por técnicas y estrategias como la reestructuración cognitiva; la autocaracterización; la técnica de la Rejilla de concentración y Stroop; habilidades de comunicación y de autoconocimiento; auto-registros conductuales; observaciones-confrontaciones e interpretaciones; y técnicas de relajación y visualización. Se administró el Cuestionario de Características Psicológicas relacionadas con el Rendimiento Deportivo en jugadores de fútbol (CPRD-f, Olmedilla, García y Martínez, 2007) antes y después de la intervención de 4meses de duración. Los resultados indican que las habilidades psicológicas en estas deportistas son caracterizadas por niveles altos de autoconfianza; impermeabilidad a la evaluación del rendimiento; manejo de la ansiedad competitiva y de la atención-concentración. Aparecen diferencias significativas tras la intervención que favorecen la calma ante las diferentes situaciones relacionadas con la competición, con un aumento del manejo de la ansiedad competitiva. Se plantea la necesidad de transferir esta información a psicólogos del deporte que intervengan a jugadoras de hockey, facilitando la selección y ajuste de estrategias psicológicas específicas en los planes de entrenamiento con el objetivo de mantener y fortalecer sus habilidades psicológicas llegando a conseguir que se manifiesten al máximo nivel.
\end{abstract}

Palabras clave: entrenamiento psicológico, hockey femenino, CPRD-f, rendimiento deportivo.

\section{ABSTRACT}

The purpose of this pilot study was to evaluate the psychological skills of grass hockey players, and, on the other hand, the effectiveness of participation in a psychological training program on psychological characteristics related to athletic performance. Ten hockey players from 16 to 26 years old participated in the senior women's field hockey team. The psychological intervention was 


\section{Entrenamiento psicológico en hockey hierba femenino}

carried out during 16 sessions through individualized work (voluntary) and group. The intervention consisted of techniques and strategies such as cognitive restructuring; self-characterization; the technique of the concentration grid and Stroop; communication and self-knowledge skills; behavioral self-records; observations-confrontations and interpretations; and relaxation and visualization techniques. The CPRD-f (Olmedilla, García and Martínez, 2007) was administered before and after the 4-month intervention. The results indicate that the psychological skills in these athletes are characterized by high levels of self-confidence; impermeability to performance evaluation; management of competitive anxiety and attention-concentration. Significant differences appear after the intervention that favor calm against the different situations related to the competition. The need arises to transfer this information to sports psychologists who intervene hockey players, facilitating the selection and adjustment of specific psychological strategies in training plans with the aim of maintaining and strengthening their psychological skills, getting them to manifest themselves to the maximum level.

Keywords: Psychological training, female hockey, CPRD-f, sports performance.

\section{RESUMO}

O objetivo deste estudo de caso foi avaliar as habilidades psicológicas de jogadoras de hóquei na grama, assim como a efetividade durante a participação em um programa de treinamento baseado nas características psicológicas relacionadas com o desempenho esportivo. Dez jogadoras de hóquei entre 16 e 26 anos participaram da seleção feminina ha categoria sênior. A intervenção psicológica foi realizada em Montevidéu, Uruguai, por meio de 16 sessões, através de trabalho individualizado (voluntário) e trabalho em grupo (obrigatório) com uma abordagem cognitiva/comportamental. A intervenção foi realizada por técnicas com o emprego de estratégias como reestruturação cognitiva, autocaracterização, técnica da capacidade de concentração e habilidades de comunicação e autoconhecimento, registros autocomportamentais, observações, confrontos e interpretações e técnicas de relaxamento e visualização. Foi aplicado um questionário das características psicológicas relacionadas ao desempenho esportivo em jogadores de futebol (CPRD-f, Olmedilla, García y Martínez, 2007) antes e após a intervenção de quatro meses. Os resultados indicam que as habilidades psicológicas desses atletas são caracterizadas por altos níveis de autoconfiança e permeabilidade à avaliação do desempenho da gestão competitiva da ansiedade e concentração. Diferenças significativas apareceram após a intervenção que favorecem a calma diante das diferentes situações relacionadas à competição, com um aumento no gerenciamento da ansiedade competitiva. Surge a necessidade então de transferir essas informações aos psicólogos esportivos que intervêm junto aos jogadores de hóquei, facilitando a seleção e o ajuste de estratégias específicas nos planos de treinamento como objetivo de manter e fortalecer suas habilidades psicológicas, fazendo com que elas se manifestem no mais alto nível.

Palavras-chave: Treinamento psicológico, hóquei feminino, CPRD-f, desempenho esportivo.

\section{INTRODUCCION}

En los últimos años ha aparecido un creciente interés por el entrenamiento psicológico (Abenza, González, Reyes, Reyes, Blas, y Olmedilla, 2014; Olmedilla, García-Mas y Ortega, 2017), cuya pretensión es la detección y evaluación de necesidades; y la planificación y desarrollo del entrenamiento de habilidades psicológicas relevantes en este contexto. Se han descrito técnicas de intervención; bases teóricas en las que se asientan dichas técnicas; y estadísticas y procedimientos metodológicos adecuados para la intervención; pero como señala Córdoba (2015), es menos frecuente que se 


\section{Tutte, Reche y Álvarez}

analicen los procesos de las intervenciones psicológicas en el ámbito deportivo y sus niveles de eficacia.

Es sabido que la identificación de los principales factores que inciden en el rendimiento de los deportistas de cada modalidad es el primer paso para diseñar una estrategia de entrenamientos específicos que mejore la efectividad del trabajo desarrollado por un deportista (Castilla y Ramos, 2012).En todas las áreas de funcionamiento que implica el deporte de competición intervienen variables psicológicas que tienen una notable importancia (autoconfianza, ansiedad, concentración), y el conocimiento de estas habilidades psicológicas en los deportistas condiciona la programación de sus entrenamientos (Reche, Cepero y Rojas, 2013).

Aparecen distintos estudios que refieren evaluaciones sobre variables psicológicas en deporte de competición en distintos deportes, en esgrima (Reche, Cepero y Rojas, 2012), en fútbol (Olmedilla, Ortega. Andreu y Ortín, 2010), en judo (Gimeno, Buceta, y PérezLlanta, 2001), en gimnastas de rítmica (Jaenes, Carmona, y Lopa, 2010), en deportes de raqueta y pala (Godoy, Vélez, y Pradas, 2009).Siendo uno de los instrumentos más seleccionados y utilizado con frecuencia el CPRD (Arias, Cardoso, Aguirre-Loaiza y Arenas, 2016; Cantón y Checa, 2011; Gimeno, Buceta y Pérez-Llanta, 2001), el CPRD-F. (López-López, Sánchez, y Vélez, 2013), el PARP1 (Serrato, García y Rivera, 2000), el PARP1-R (Serrato, 2006); o el OMSAT-3 (Durand-Bush, Salmela, y GreenDemers, 2001).

En cuanto a intervenciones psicológicas dentro del ámbito deportivo, se han descrito numerosas intervenciones con terapias emergentes de tercera generación con niveles de eficacia. Es así que, mediante mindfulness se han obtenido mejoras en atletas que favorecían distintos estados de ansiedad y concentración (Hoja \& Jansen, 2019). Sin embargo, se observan también mejoras con tratamientos o estrategias más clásicos de segunda generación, basadas en las terapias cognitivo-conductuales (Olmedilla, Ortega, Andreu y Ortín, 2010).

En el caso del hockey no se encuentra demasiada evidencia científica acerca de evaluaciones sobre habilidades psicológicas y su dominio, ni en cuanto a intervenciones psicológicas. En este último caso, se han descrito mejoras en el rendimiento mediante intervenciones psicológicas basadas en mindfulness para favorecer distintos estados de ansiedad y concentración (Buhlmayer, Birrer, Rothlin et al, 2017); con intervenciones en autoestima (Mikhnov, 2015); mediante autodiálogo (Imagawa, ySakuma, 2014; Nippert y Smith, 2008) y trabajo de relajación (Rogerson, y Hrycaiko, 2002); y favoreciendo estrategias de afrontamiento de manejo del estrés mediante reducción ansiedad y aumento de la autoestima (Kaplanova, 2019).

Para que una intervención psicológica sea competente, el psicólogo deportivo debe analizar las necesidades del cliente; evaluar y diagnosticar; delimitar objetivos; generar confianza; definir la intervención y planificarla; $y$ por último señalar los indicadores que se van a utilizar en la medida de la eficacia del tratamiento (Córdoba, 2015).En definitiva, la valoración del trabajo psicológico debe centrarse, en la mejora del funcionamiento psicológico de los deportistas, valorando su progreso adaptativo, su relación con el rendimiento y su bienestar psicológico (Kosendiak y Ptak, 2017;Olmedilla y Dominguez-Igual, 2016).

El objetivo de estudio es identificar el perfil psicológico de jugadoras de hockey de alto rendimiento mediante una evaluación de habilidades psicológicas que permita al psicólogo implementar programas de entrenamiento para mantenerlas y fortalecerlas; y determinar estrategias de intervención adecuadas en aquellas que requieran seguimiento. Además, y con la finalidad de observar si tras la intervención 


\section{Entrenamiento psicológico en hockey hierba femenino}

aparece una mejora en las habilidades psicológicas que intervienen en el rendimiento deportivo de jugadoras de hockey hierba, se evalúa subjetivamente el grado de impacto del entrenamiento de las mismas tras un programa de preparación mental.

\section{METODOLOGIA}

Se trata de una investigación empírica, que utiliza la estrategia manipulativa. Específicamente este trabajo refiere a un diseño cuasi-experimental (Ato, López y Benavente, 2013). Hay manipulación de la variable independiente: programa de intervención, pero queno cumple con el criterio de la asignación al azar de las participantes.

\section{Participantes}

El estudio se llevó a cabo con 10 jugadoras de hockey hierba, integrantes de la selección uruguaya de hockey hierba, con edades comprendidas entre los16 y 26 años (edad: $M$ $=21, D T=3,71)$ y en periodo de entrenamiento precompetitivo, preparatorio para la clasificación al preolímpico (octubre a febrero), con 6 sesiones de entreno semanales. El tipo de muestreo utilizado es no probabilístico, por conveniencia. Resultando central que las participantes cumplieran con el criterio de haber asistido al menos al $80 \%$ de las sesiones de este programa de intervención psicológica.

El objetivo del programa es la identificación y optimización de habilidades psicológicas relacionadas con el alto rendimiento, tomando en cuenta las características psicológicas de un deporte colectivo. El cuerpo técnico está formado por 2 técnicos, 1 preparador físico, 1 fisioterapeuta, 1médico, 1 nutricionista y 1 psicólogo.

\section{Instrumentos}

Se aplicó el Cuestionario de Características Psicológicas relacionadas con el Rendimiento
Deportivo en jugadores de futbol (CPRD-f; Olmedilla, Garcia y Martìnez, 2007). Este procede de la versión española del cuestionario original Psychological Skills Inventory for Sports (PSIS) de Mahoney (1989), validada por Gimeno, Buceta y PérezLlantada (2001), y cuyo objetivo era evaluar una serie de habilidades psicológicas implicadas en el rendimiento en el deporte de competición. El cuestionario consta de 4 factores y 29 ítems: autoconfianza (10 ítems), influencia de la evaluación del rendimiento (8 ítems), ansiedad (5 ítems) y concentración (6 ítems). Para las respuestas, usa una escala Líkert de 5 alternativas de respuesta que va de Totalmente de Acuerdo a Totalmente en Desacuerdo, más la opción adicional para los casos en los que el futbolista "no entiende el ítem" (Gimeno, Buceta, Pérez-Llantada, 2001). La autoconfianza alta está determinada por puntuaciones entre 20 y 40 , la baja entre 0 y 19. La influencia de la evaluación alta entre 16 y 32 y baja entre 0 y 15 . La ansiedad alta de 10 a 20 y la baja de 0 a 9 . Finalmente, la concentración alta de 12 a 24 y la baja de 0 a 11.

En la escala autoconfianza, se incluyen ítems que evalúan diferentes aspectos de la ansiedad cognitiva, del manejo de la atención durante la competición y del manejo de la tensión (ansiedad somática). Una puntuación alta indica que el deportista tiende a mostrar una confianza muy buena en sus recursos para controlar el estrés potencial de la competición. Una puntuación baja, indica que el deportista no tiene muchos recursos para enfrentar el estrés que supone la competición $\mathrm{y}$ esto puede repercutir negativamente en su desempeño.

La escala de influencia de evaluación de rendimiento se centra específicamente en el impacto que tienen situaciones estresantes características de la competición deportiva, en su mayoría relacionadas con el rendimiento del deportista y la evaluación del mismo por parte de otras personas o del propio deportista, así como de la evaluación del rendimiento ante errores en competición. Una 


\section{Tutte, Reche y Álvarez}

puntuación alta en esta escala, indica que al deportista no le afectan negativamente estas situaciones. Una puntuación baja, indica que este deportista es permeable a la evaluación que los otros hacen de su rendimiento.

En lo que respecta a la ansiedad competitiva, se incluyen ítems relacionados con diferentes aspectos de la ansiedad competitiva; ansiedad respecto a la preparación de la competición, a los momentos previos y durante la competición. Una puntuación alta en esta escala, indica que el deportista no siente ansiedad ante las diferentes situaciones relacionadas con la competición. Una puntuación baja, indica que el deportista si incertidumbre y duda frente a las situaciones que se pueden generar en la competición.

En referencia a la concentración, se incluyen los ítems relacionados con diferentes aspectos del manejo de la atención durante la competición. Una puntuación alta en esta escala, indica que el deportista maneja con garantías el enfoque atencional atendiendo a lo que realmente debe atender. Una puntuación baja en esta escala, indica que el deportista tiende a distraerse, atendiendo a estímulos poco influyentes en la competición $\mathrm{y}$ en el rendimiento.

Se ha considerado la utilización de este cuestionario por su utilidad para la evaluación del perfil psicológico de los deportistas, aludiendo a habilidades psicológicas que caracterizan el alto rendimiento.

\section{Procedimiento}

La intervención fue solicitada por el entrenador, en el marco de una serie de torneos clasificatorios para obtener un cupo en el pre-olímpico. Las necesidades explicitadas por el entrenador hacían referencia a la identificación y optimización de habilidades psicológicas relacionadas con el alto rendimiento y con la regulación de las emociones, tomando en cuenta las características psicológicas de un deporte colectivo y femenino.

Los criterios de exclusión en nuestro estudio fueron no haber participado en más del $80 \%$ de las sesiones del programa psicológico y pruebas con falta de cumplimentación de datos esenciales y/o ítems necesarios tanto en el pre test como en el pos test. El procedimiento fue aprobado por el comité de ética de la Universidad Católica San Antonio de Murcia.

A partir de esta petición, elaboramos un programa de intervención, con una extensión de 16 sesiones, con una frecuencia semanal, de una duración de 60 a 80 minutos y una duración total de 4 meses de trabajo individualizado (voluntario) y trabajo grupal (obligatorio) con un enfoque cognitivoconductual. La intervención estuvo formada por técnicas y estrategias como la reestructuración cognitiva; la autocaracterización; la técnica de la Rejilla de concentración y Stroop; habilidades de comunicación y de autoconocimiento; autoregistros conductuales; observacionesconfrontaciones e interpretaciones; y técnicas de relajación y visualización.

La preparación mental siguió la siguiente secuencia: evaluación o caracterización psicológica inicial; identificación del perfil psicológico; intervención psicológica; explicación, aprendizaje y aplicación de técnicas y estrategias; evaluación psicológica final; y valoración de la eficacia del programa $\mathrm{y}$ recomendaciones de futuro.

\section{Estructura de las sesiones}

La primer y segunda sesión estuvieron destinadas a la explicación los objetivos de la intervención y a recabar los consentimientos informados que evidenciaran la voluntariedad de las participantes de integrar dicho programa. Posteriormente, se procedió a la administración del cuestionario de 


\section{Entrenamiento psicológico en hockey hierba femenino}

Características Psicológicas relacionadas con el Rendimiento Deportivo en jugadores de futbol (CPRD-f; Olmedilla, García y Martínez, 2007), en formato de auto-reporte yen una sesión colectiva, en el mismo lugar de entrenamiento, previo al entrenamiento técnico -táctico, que se realizaba durante la tarde (18 horas. aproximadamente) y que se desarrolló entre los meses de octubre y febrero, correspondiendo a las estaciones de primavera-verano. Se explicitó a las deportistas el carácter confidencial de los datos recogidos.

A partir de esta primera instancia de evaluación (pre) y administración del cuestionario, nos planteamos una serie de objetivos que son los que guiaron la intervención. El objetivo central estaba orientado a identificar la presencia de habilidades psicológicas deportivas (autoconfianza, influencia de evaluación de rendimiento, ansiedad competitiva $y$ concentración. Una vez identificados los niveles en los que se encontraban las deportistas en relación a estas habilidades, nos planteamos un segundo objetivo, que estuvo dirigido a elaborar un plan de intervención que optimizara sus habilidades psicológicas para una mejora en el rendimiento deportivo de las atletas.

Una vez, obtenidos los resultados de los cuestionarios en la instancia pre, se le realizó a cada deportista una devolución individual, explicando las puntuaciones obtenidas y el significado de las mismas. La duración de esa entrevista fue entre 20 y 30 minutos, una semana después de la administración de los cuestionarios. Estas sesiones individuales llevaron una semana de trabajo y se realizó exactamente igual, luego de la segunda evaluación. Siendo las únicas instancias de carácter individual. El resto de las sesiones fueron grupales.

La tercera sesión estuvo dirigida al trabajo de la autoconfianza, a partir del reconocimiento de las fortalezas y las debilidades (lo denominamos aspectos a mejorar) tanto individuales como colectivos que estaban presentes en el equipo. Cada deportista se tomó unos minutos para poder pensar y redactar la consigna indicada. Luego se puso en común y aquellas fortalezas que aparecían con más frecuencia, fueron las que se tomaron como características del equipo. Luego se hizo lo mismo con las debilidades $\mathrm{y}$, por último, se concluyó cual era el porcentaje en que se utilizaban esas fortalezas y debilidades seleccionadas. Esto quedó redactado y se colgó en el vestuario, manteniéndose a lo largo de todo el tiempo que implicó la intervención.

Las sesiones 4 y 5: profundizaron los conceptos de atención-concentración a partir de una conferencia a modo de taller, donde se exponían las características de estos procesos cognitivos, su manifestación en el deporte, especificando las propiedades de las mismas en un deporte colectivo de oposición. Posteriormente, se utilizaron dos técnicas para su entrenamiento: una fue el test de Rejilla (Harris y Harris, 1992) y la segunda el uso del test de efecto Stroop, que evalúa y entrena la interferencia semántica, y es muy utilizado como recurso para trabajar la concentración en el deporte. En la sesión 5, se le solicitó al entrenador, que incorporara al entrenamiento físico, técnico y táctico el uso de materiales de diversos colores, sonidos y que buscara la superposición de estímulos, para trabajar la focalización.

Las sesiones 6, 7 y 8: estuvieron centradas en la ansiedad precompetitiva. En la sesión 6 se presentó teóricamente a esta variable, explicando sus componentes y características. Se determinaron sus tres componentes y las herramientas para poder ser entrenados.

En la sesión 7, se trabajó en el primer componente: la ansiedad cognitiva, a través de la parada de pensamiento y posterior reestructuración cognitiva, se le solicitó a cada deportista que identificaran los posibles pensamientos amenazantes que pudieran surgir el día previo a competir. Una vez que las deportistas tenían claridad en sus 


\section{Tutte, Reche y Álvarez}

pensamientos, se les pidió que crearan un pensamiento positivo para cada pensamiento negativo que habían identificado previamente. Por último, se motivó a encontrar una acción física (p.ej. aplauso, salto rápido), que pudiera motivar a eliminar ese pensamiento amenazante que podría repercutir negativamente en el rendimiento.

En la sesión 8, se hizo hincapié en la ansiedad somática, es decir las repercusiones en el cuerpo que pueden aparecer los días antes de la competición. Para poder controlar esta ansiedad, se llevó a cabo una técnica de relajación muscular progresiva breve de Jacobson. Se realizó de forma colectiva, en colchonetas individuales en el mismo lugar de entrenamiento.

La sesión 9 y 10: estuvo dirigida a trabajar la habilidad comunicación, para ello realizamos una presentación de esta variable a través de videos e imágenes, para poder determinar la comunicación verbal y no verbal y los componentes que integran a cada una.

Llevamos esto al entrenamiento, donde limitamos la posibilidad de hablar, en búsqueda de otras formas de poder comunicarse y aprender a tomar decisiones colectivas sin hacer verbalizaciones.

En la décima sesión, abordamos la comunicación frente al error y la adversidad, y analizamos las formas en que comunicamos nuestra frustración al propio equipo y a los rivales.

Las sesiones 11, 12, 13 y 14: abordamos la temática de las emociones. Comenzamos por identificar las emociones negativas que se presentaban en el equipo y como hacíamos frente a las mismas. Se les brindó información de la temporalidad de las emociones, de la cantidad de emociones que existían y decidimos categorizarlas en poco frecuentes, frecuentes y muy frecuentes. Así, se obtuvo claridad de cuáles eran las emociones que caracterizaban al equipo.
En la sesión 12, se presentó teóricamente las habilidades optimismo y resiliencia, y la función de ambas como variables protectoras y canalizadoras de las emociones. A través de esos conceptos, pudimos ahondar en las situaciones de adversidad y encontrar las estrategias de afrontamientos con las que contaba el equipo. A su vez, si las mismas resultaban efectivas o no.

En la sesión 13, realizamos una técnica de visualización, atravesando distintas emociones y situaciones que este equipo ya había experimentado en diferentes campeonatos.

Por último, en la 15 sesión: llevamos adelante otra técnica de visualización, orientada a experimentar la sensación de bienestar y disfrute a partir de emociones placenteras y de confianza, con miras al inicio del torneo clasificatorio a un preolímpico.

Finalmente, en la sesión 16, realizamos la segunda instancia de evaluación (post), donde administramos nuevamente los cuestionarios con la finalidad de encontrar modificaciones en las puntuaciones, que podrían indicar la mejora o no de las habilidades trabajadas. Y posteriormente se realizaron las devoluciones individuales.

\section{Análisis de los datos}

Se realizaron análisis descriptivos (media, y desviación típica) de las puntuaciones totales de cada uno de los factores del cuestionario (autoconfianza, influencia de la evaluación del rendimiento, ansiedad y concentración). Se realizaron análisis para comprobar la normalidad de la distribución de la muestra con la prueba de Shapiro-Wilk, obteniendo resultados normales para todas las subescalas que conforman el cuestionario, pero debido al tamaño muestral $(\mathrm{n}<30)$, no se pudo hacer uso de pruebas paramétricas. Por ello, para apreciar las posibles diferencias estadísticamente significativas en las puntuaciones antes del comienzo del programa de intervención, y después del 


\section{Entrenamiento psicológico en hockey hierba femenino}

mismo, se aplicó la prueba t Wilcoxon para muestras apareadas con un nivel de significación de p>.05

Se utilizó el paquete estadístico SPSS, versión 25 , para la subida y análisis de los datos.

\section{RESULTADOS}

Tanto las deportistas como el cuerpo técnico, expresaron a través de una encuesta abierta la satisfacción del programa, mencionando como principales aportes, el contar con un espacio para poder hablar y reflexionar de las situaciones que experimentaban individual y colectivamente, así como de aprender herramientas para poder controlar la ansiedad y las emociones. Manifestaron sentir disfrute por las tareas, señalando la relajación y visualización como las técnicas preferidas.

El perfil psicológico de las deportistas se define por puntuaciones elevadas en todas las dimensiones del cuestionario, en autoconfianza, en influencia de la evaluación del rendimiento, ansiedad competitiva y concentración. En la Tabla 1 se pueden ver las puntuaciones obtenidas en las jugadoras en los factores antes de la intervención psicológica. Las medias obtenidas se encuentran dentro de los resultados esperados para cada subescala.

Tabla.1 Resultados del CPRD-f antes

\begin{tabular}{lccclc}
\hline & \multicolumn{5}{c}{ Medida Inicial } \\
\cline { 2 - 6 } A & 27,30 & 3,65 & 29 &,$- 62 /, 714$ & Shapiro-Wil-P \\
Inf & 18,50 & 3,27 & 19 &,$- 25 /-, 201$ &, 70 \\
Ans & 12,00 & 2,45 & 9 &, $00 /-, 12$ &, 46 \\
Con & 15,70 & 2,00 & 15 &,$- 11 /, 35$ &, 29 \\
\hline A: Autoconfianza; Inf: Influencia Evaluación Negativa; Ans: Ansiedad; Con: Concentración \\
*p p 0.05
\end{tabular}

La Tabla 2 presenta los resultados de las subescalas del CPRD-f después del programa y tras el mismo encontramos que las medias estadísticas son superiores en la dimensión ansiedad y concentración; e inferiores en autoconfianza, e influencia de la evaluación del rendimiento. Encontrándose diferencias significativas en la ansiedad competitiva.
Tabla.2 Resultados del CPRD-f después de la intervención. Análisis de diferencias significativas

\begin{tabular}{lcccccc}
\hline & \multicolumn{5}{c}{ Medida Final } \\
\cline { 2 - 7 } & Media & D.T. & Moda & Asimetrí/Curtosis & Shapiro-Wilk & Wilcoxon-P \\
A & 26,90 & 6,66 & 28 &,$- 972 /, 061$ &, 703 & 1 \\
Inf & 17,50 & 4,19 & 17 &, $902 / 360$ &, 459 &, 59 \\
Ans & 14,60 & 2,01 & 14 &,$- 541 /-, 495$ &, 296 &, $01^{*}$ \\
Con & 17,20 & 4,37 & 15 & $, 312 /-1,14$ &, 908 &, 23 \\
\hline A: Autoconfianza; Inf: Influencia Evaluación Negativa; Ans: Ansiedad; Con:Concentración \\
"p significativa si p < . 05
\end{tabular}

Para completar esta información se realizó un análisis de los ítems y se hallaron 2 ítems con diferencias estadísticamente significativas. En la Tabla 3 se pueden ver las puntuaciones obtenidas en estos ítems, ambos del factor Ansiedad.

Tabla.3 Puntuaciones en los ítems del CPRD antes y después del entrenamiento psicológico

\begin{tabular}{|c|c|c|c|c|c|c|}
\hline \multirow[b]{2}{*}{ ANSIEDAD } & \multirow[b]{2}{*}{ CONTENIDO DEL ITEM } & \multicolumn{2}{|c|}{ Medida Inicial } & \multicolumn{2}{|c|}{ Medida Final } & \multirow[t]{2}{*}{ P valor } \\
\hline & & Media & D.T. & Media & D.T. & \\
\hline ITEM 11 & $\begin{array}{l}\text { Algunas veces siento una } \\
\text { intensa ansiedad mientras } \\
\text { estoy participando en una } \\
\text { prueba (o partido) }\end{array}$ & 1.5 & .70 & 2.6 & .51 & $.008^{*}$ \\
\hline ÍTEM 21 & $\begin{array}{l}\text { El día anterior a una } \\
\text { competición (o partido) me } \\
\text { encuentro habitualmente } \\
\text { demasiado nervios@o } \\
\text { preocupad@ }\end{array}$ & 2.9 & .87 & 3.6 & .51 & $.035^{*}$ \\
\hline
\end{tabular}

\section{DISCUSIÓN}

El presente estudio tuvo como objetivo analizar el perfil psicológico de jugadoras de hockey de alto rendimiento, aumentar sus habilidades psicológicas en sus entrenamientos y competición a través de un programa de entrenamiento psicológico, y evaluar los cambios. La evaluación de las habilidades psicológicas tiene por objeto mejorar el rendimiento deportivo (Abenza et al., 2014; Olmedilla, Fuensanta, Almansa, Gómez-Espejo, y Ortega, 2018) y es a partir de la identificación de este perfil en la etapa pre y la implementación de una intervención, que tuvimos la intención de poder evaluar las modificaciones que este programa puede haber generado.

La evaluación con el CPRD-f mostró un 


\section{Tutte, Reche y Álvarez}

perfil, en las integrantes de la selección uruguaya de hockey hierba, con niveles altos de confianza, relacionados con recursos para controlar el estrés potencial de la competición. Además, impermeabilidad a la evaluación del rendimiento o bajo impacto de las situaciones estresantes características de la competición deportiva, en su mayoría relacionadas con el rendimiento y la evaluación del mismo por parte de otras personas o del propio deportista, así como de la evaluación del rendimiento ante errores en competición. Estos resultados coinciden con los obtenidos por (Andrade-Sánchez, Galindo-Villardón y Cuevas Romo, 2015) donde los practicantes de algún deporte individual tienen un menor control de estrés en relación a aquellos que practican alguna modalidad colectiva. Y se confirman con los resultados arrojados en una investigación con jugadoras de fútbol (Olmedilla, et al., 2018) dónde aparece un adecuado control del estrés que mejora a partir de la implementación de una intervención psicológica.

El perfil de las jugadoras arrojó también un adecuado manejo de la ansiedad, tanto en la preparación de la competición, como en los previos y durante la competición; así como un adecuado umbral de la atención-concentración en competición. Perfil adecuado y esperado en estas deportistas de alto nivel, pero con capacidad de mejora. Resultados similares de encontraron en un estudio que nuclea a jugadores de fútbol, judokas y nadadores (Gimeno, Buceta y Pérez- Llantada, 2007).

Este perfil complementa información acerca de variables psicológicas con otro estudio que encuentra puntuaciones elevadas en motivación y confianza en jóvenes jugadores de hockey hierba élite (Elferink-Gemser, Visscher, Lemmink, y Mulder, 2007). Motivación más elevada en la élite que en la no elite (Elferink-Gemser, Visscher, Lemmink, y Mulder, 2004). Además, con otros estudios de hockey, aunque en este caso sobre hielo, que caracterizan las funciones ejecutivas (Lundgren et al., 2016);o que describen como jugadores experimentados adultos presentan un mejor control bajo estrés y niveles más bajos de ansiedad que jugadores más jóvenes (Géczi et al., 2008),estos últimos sin diferencias en determinados grupos de edad, en ansiedad, estrategias de afrontamiento, y personalidad (Géczi, Tóth, Sipos, Fügedi, Dancs, y Bognár, 2009).

Tras el entrenamiento psicológico de 4 meses de duración desde una perspectiva cognitivoconductual, y con la mayor parte de las sesiones centradas en desarrollar sensaciones de calma frente la ansiedad competitiva y técnicas de restructuración cognitiva frente a los pensamientos amenazantes; acompañadas de sesiones de autocaracterización; de técnica de la Rejilla de concentración y Stroop; de habilidades de comunicación y de autoconocimiento; auto-registros conductuales; observaciones-confrontaciones e interpretaciones; y técnicas de relajación y visualización; se consiguen bajar los niveles de ansiedad de las deportistas, consiguiendo que gestionen mejor el estrés propio de competición y del entrenamiento. En relación a los ítems que evidencian diferencias estadísticas, y que integran el factor ansiedad vemos que hacen referencia a verbalizaciones que aluden a la ansiedad durante una competición y en los momentos previos de la misma.

Por ende, y siguiendo la evidencia científica, con la disminución de la ansiedad podemos augurar un mejor rendimiento (Butt, Weinberg, y Horn,2003) y una mejoría en las estrategias de afrontamiento más óptimas (Kaplánová, 2019).

Además, aunque de forma no significativa, se consigue aumentar la concentración y atención en lo relevante, sin un anclaje en los errores y con una reinterpretación de los mismos, percibiéndolos como algo natural de la práctica deportiva. En la línea de lo conseguido con un programa de intervención en futbolistas con algunas de las técnicas utilizadas bajo el enfoque cognitivoconductual para la dimensión de concentración (Olmedilla y Dominguez-Igual, 


\section{Entrenamiento psicológico en hockey hierba femenino}

2016)

La falta de cambios debidos al programa de entrenamiento psicológico en el resto de dimensiones del cuestionario pudiera ser porque las puntuaciones medias al inicio eran altas, con lo que resulta muy difícil experimentar aumentos que permitan obtener significaciones estadísticas; y el tiempo de entrenamiento psicológico (de 4 meses, distribuidos en 16 sesiones) quizá haya sido insuficiente para poder observar mejoras reales.

El nivel de satisfacción con el entrenamiento psicológico tanto por parte de los deportistas como por el cuerpo técnico fue elevado, manifestando sentir disfrute y aprendizaje, destacando como técnicas de preferencia la relajación y la visualización. Además, agradeciendo el haber podido generar un espacio para el intercambio y la experimentación, a través de una encuesta de satisfacción adhoc. Resultados similares fueron encontrados con deportistas olímpicos polacos (Kosendiak y Ptak, 2017) aludiendo al agrado por la elección de las técnicas de relajación y visualización, dentro de un programa de intervención psicológica.

Tanto los datos obtenidos, como la propuesta de intervención, podrían resultar como una herramienta útil al psicólogo deportivo tanto para desarrollar hipótesis de trabajo, como intervenciones más adecuadas a sus jugadoras de hockey.

Como propuestas de futuro observamos necesario establecer protocolos de identificación de habilidades psicológicas en los distintos deportes, utilizándolos como recursos para establecer hipótesis de trabajo acerca de la intervención psicológica más apropiada para el aumento del rendimiento deportivo, así como difundir nuestra propuesta de intervención para ajuste y desarrollo de aspectos del programa en la selección de estrategias de intervención de psicólogos del deporte.

\section{Limitaciones}

Como limitaciones podemos señalar que se trata de datos autoinformados y que los estudios previos en hockey, específicamente sobre hierba, son realmente escasos.

\section{CONCLUSIONES}

De los resultados mostrados, de su análisis y de su discusión, se pueden obtener las siguientes conclusiones, sobre las habilidades psicológicas de jugadoras de hockey hierba uruguayas antes y después de una preparación mental inmersa en un programa de entrenamiento psicológico para la preparación del preolímpico con el fin de aumentar el rendimiento de las atletas:

1) Las habilidades psicológicas de las deportistas previa intervención se caracterizan por niveles elevados en autoconfianza a la hora de controlar el estrés de la competición; impermeabilidad a la evaluación del rendimiento en competición tanto por su parte como por parte de personas ajenas; manejo de la ansiedad en los entrenos, previos y durante la competición; y manejo de la atenciónconcentración en competición. Se trata de un perfil psicológico óptimo, característico del alto rendimiento, pero con capacidad de mejora.

2) Tras la intervención psicológica cognitivo conductual, a lo largo de 4 meses y 16 sesiones, con técnicas y estrategias como autocaracterización; la técnica de la Rejilla de concentración y Stroop; de habilidades de comunicación y de autoconocimiento; auto-registros conductuales; observacionesconfrontaciones e interpretaciones; y técnicas de relajación y visualización, se alcanzan una mejor gestión del estrés propio de competición y del entrenamiento, con reportes de satisfacción con lo aprendido e integrado. 


\section{Tutte, Reche y Álvarez}

3) Se requiere de más investigación clínica en el ámbito de la psicología del deporte con intervenciones psicológicas y niveles de eficacia en hockey hierba femenino.

\section{REFERENCIAS}

Abenza, L., González, J., Reyes, L., Reyes, F., Blas, A. y Olmedilla, A. (2014). Descripción y evaluación del entrenamiento psicológico de una deportista de regata clase laser radial. Revista Iberoamericana de Psicología del Ejercicio y el Deporte, 9(1), 67-92.

Álvarez, O., Falco, C., Estevan, I., MolinaGarcía, J., y Castillo, I. (2013). Intervención psicológica en un equipo de gimnasia rítmica deportiva: Estudio de un caso. Revista de Psicología del Deporte, 22(2), 395-401.

Andrade-Sánchez, A., Galindo-Villardón, M., y Cuevas Romo, J. (2015) Análisis multivariante del perfil psicológico de los deportistas universitarios. Aplicación del CPRD en México. Educación Física y Ciencia, 17 (2), 1-11.

Arias, I., Cardoso, T., Aguirre, H. y Arenas, J. (2016). Características psicológicas de rendimiento deportivo en deportes de conjunto. Psicogente, 19(35), 25-36. http://doi.org/10.17081/psico.19.35.1206

Ato, M., López, J. y Benavente, A. (2013). Un sistema de clasificación de los diseños de investigación en psicología. Anales de Psicología, $29 \quad$ (3), 1038-1059. http://dx.doi.org/10.6018/analesps.293.17851 1

Bühlmayer, L., Birrer, D., Röthlin, P., Faude, O., y Donath, L. (2017). Effects of mindfulness practice on performance-relevant parameters and performance outcomes in sports: A meta-analytical review. Sports Medicine, 47(11), 2309-2321.
Butt, J., Weinberg, R., y Horn, T. (2003). The intensity and directional interpretation of anxiety: Fluctuations throughout competition and relationship to performance. The Sport Psychologist, 17(1), 35-54.

Fenoy Castilla, J., y Campoy Ramos, L. (2012). Rendimiento deportivo, estilos de liderazgo y evitación experiencial en jóvenes futbolistas almerienses. Revista Psicología del Deporte, 21 (1), 137-142.

Cantón Chirivella, E. y Checa Esquiva, I. (2011) Entrenamiento psicológico en baile deportivo y de competición.Revista de Psicología del Deporte, 20 (2), 479-490.

Córdoba, E. P. (2013). Análisis teórico del proceso de intervención psicológica en el deporte. Cuadernos de Psicología del Deporte, 13(2), 113-124.

Durand-Bush, N., Salmela, J. H., y GreenDemers, I. (2001). The Ottawa mental skills assessment tool (OMSAT-3*). The Sport Psychologist, 15(1), 1-19.

Elferink-Gemser, M. T., Visscher, C., Lemmink, K. A., y Mulder, T. (2007). Multidimensional performance characteristics and standard of performance in talented youth field hockey players: A longitudinal study. Journal of sports sciences, 25(4), 481489.

Elferink-Gemser, M., Visscher, C., Lemmink, K., y Mulder, T. (2004). Relation between multidimensional performance characteristics and level of performance in talented youth field hockey players. Journal of sports sciences, 22(11-12), 1053-1063.

García, C. R., González, M. C., y Ruiz, F. J. R. (2012). Perfil psicológico en esgrimistas de alto rendimiento. Cultura, Ciencia y Deporte, 7(19), 35-44.

Géczi, G., Bognár, J., Tóth, L., Sipos, K., y Fügedi, B. (2008). Anxiety and coping of Hungarian national ice hockey players. International Journal of Sports Science and 


\section{Entrenamiento psicológico en hockey hierba femenino}

Coaching, 3, 277-285.

Géczi, G., Tóth, L., Sipos, K., Fügedi, B., Dancs, H., y Bognár, J. (2009). Psychological profile of Hungarian national young ice hockey players. Kinesiology: International journal of fundamental and applied kinesiology, 41(1), 88-96.

Gimeno, F. y Guedea, J. A. (2001). Evaluación e intervención psicológica en la "promoción" de talentos deportivos en judo. Revista de psicología del deporte, 10, (1).

Gimeno, F., Buceta, J. M., y Pérez-Llanta, M. D. C. (2001). El cuestionario «características psicológicas relacionadas con el rendimiento deportivo»(CPRD): Características psicométricas. Análise Psicológica, 19(1), 93113.

Gimeno, F., Buceta, J.M. y Pérez-Llantada, M. C. (2001). El cuestionario "Características Psicológicas relacionadas con el Rendimiento Deportivo" (CPRD): Características psicométricas. Análise Psicológica, (19), 93-113.

Gimeno, F., Buceta, J. M. y Pérez-Llantada, M. (2007). Influencia de las variables psicológicas en el deporte de competición: Evaluación mediante el cuestionario de características psicológicas relacionadas con el rendimiento deportivo. Psicothema, 19 (4), 667-672.

Godoy, D., Vélez, M. y Pradas, F. (2009). Nivel de dominio de las habilidades psicológicas en jóvenes jugadores de tenis de mesa, bádminton y fútbol. Revista de Psicología del Deporte, 18 (1), 7-22.

Harris, D. V. y Harris, B. L. (1992). Psicología del deporte: integración mente сиеrpo. Barcelona: Hispano Europea.

Hoja, S., y Jansen, P. (2019). Mindfulnessbased intervention for tennis players: a quasiexperimental pilot study. BMJ Open Sport \& Exercise Medicine, 5(1), e000584.doi:10.1136/ bmjsem-2019-000584
Imagawa, S., ySakuma, H. (2014). The Effects of Self-Talk on Psychological Skill of Ice Hockey Players. International Conference on Social, Education and Management Engineering. Macao, China.

Jaenes, J. C., Carmona, J. y Lopa, E. (2010) Evaluación y análisis de habilidades psicológicas relacionadas con el rendimiento deportivo en gimnastas de rítmica. Revista Iberoamericana de Psicología del Ejercicio y el Deporte, 5 (1), 15-28.

Kaplánová, A. (2019). Self-esteem, anxiety and coping strategies to manage stress in ice hockey. Acta Gymnica, 49(1), 10-15. http://dx.doi.org/10.5507/ag.2018.026

Kosendiak J., y Ptak E. (2017). Psychological Support as a Factor of the Training Process Quality Improvement. Journal of Education, Health and Sport, 7(6), 807-818. DOI http://dx.doi.org/10.5281/zenodo.1038381

López-López, I. S., Sánchez, J. C. J., y Vélez, D. C. (2013). Adaptación para futbolistas (CPRD-F) del cuestionario "características psicológicas relacionadas con el rendimiento deportivo"(CPRD). Cuadernos de Psicología del Deporte, 13(2), 21-30.

Lundgren, T., Högman, L., Näslund, M., y Parling, T. (2016). Preliminary investigation of executive functions in elite ice hockey players. Journal of clinical sport psychology, 10(4), 324-335.

Mahoney, MJ. (1989). Psychological predictors of elite and non-elite performance in olympic weightlifting. International Journal of Sport Psychology, 20(1), 12- 23.

Mikhnov, A. P. (2015). Effectiveness of competitive activity of high class hockey players accounting a level of their selfesteem. Pedagogics, psychology, medicalbiological problems of physical training and sports, 2 , 47-52. http://dx.doi.org/10.15561/18189172.2015.02 08 


\section{Tutte, Reche y Álvarez}

Montero, I., y León, O. G. (2002). Clasificación y descripción de las metodologías de investigación en Psicología. International journal of clinical and health psychology, 2(3), 503-508.

Nieuwenhuis, C. F., Spamer, E. J., y Rossum, J. H. V. (2002). Prediction function for identifying talent in 14-to 15-year-old female field hockey players. High Ability Studies, 13(1), 21-33.

Nippert, A. H., y Smith, A. M. (2008). Psychologic stress related to injury and impact on sport performance. Physical medicine and rehabilitation clinics of North America, 19(2), 399-418.

Olmedilla, A., y Dominguez-Igual, J. J. (2016). Entrenamiento psicológico para la mejora de la atención y la autoconfianza en un futbolista. Revista de Psicología Aplicada al Deporte y al Ejercicio Físico, 1(1), 1-11.

Olmedilla, A., Fuensanta, M., Almansa, C., Gómez-Espejo, V. y Ortega, E. (2018) Entrenamiento psicológico y mejora de aspectos psicológicos relevantes para el rendimiento deportivo en jugadoras de fútbol. Revista de Psicología Aplicada al Deporte y al Ejercicio Físico, 3 (8), 1-11.

Olmedilla, A., García, C., y Martínez, F. (2007). Factores psicológicos vulnerabilidad a las lesiones deportivas: un estudio futbolistas. Revista de psicología del Deporte, 15(1).

Olmedilla, A., García-Mas, A. y Ortega, E. (2017). Características psicológicas para el rendimiento deportivo en jóvenes jugadores de fútbol, rugby y baloncesto. Acción Psicológica, $\quad$ 14(1), 7-16. https://doi.org/10.5944/ap.14.1.19249

Olmedilla, A., Ortega, E., Andreu, M. D., y Ortín, F. J. (2010). Programa de intervención psicológica en futbolistas: evaluación de habilidades psicológicas mediante el CPRD. Revista de Psicología del Deporte, 19(2), 249-262.
Reche-García, C., Cepero-González, M., y Rojas-Ruíz, F. J. (2013). Consideraciones en el entrenamiento de habilidades psicológicas en esgrima. Cuadernos de Psicología del Deporte, 13(2), 83-88.

Rogerson, L. J., y Hrycaiko, D. W. (2002). Enhancing competitive performance of ice hockey goaltenders using centering and selftalk. Journal of Applied Sport Psychology, 14(1), 14-26.

Serrato, L. H. (2006). Revisión y estandarización de la prueba elaborada para evaluar rasgos psicológicos en deportistas (PAR-P1) en un grupo de deportistas de rendimiento en Colombia. Cuadernos de Psicología del Deporte, 6 (2), 67-84.

Serrato, L. H., García, S. Y. y Rivera, J. F. (2000). PAR P1: Prueba para evaluar Rasgos Psicológicos en Deportistas (Manual). Bogotá: PAR LTDA. 